\title{
Differentiation of Monkey Embryonic Stem Cells to Hepatocytes by Feeder-Free Dispersion Culture and Expression Analyses of Cytochrome P450 Enzymes Responsible for Drug Metabolism
}

\author{
Junya Maruyama ${ }^{a}$ Tamihide Matsunaga,${ }^{b}$ Satoshi Yamaori, ${ }^{a}$ Sakae Sakamoto, ${ }^{c}$ Noboru Kamada,${ }^{c}$ \\ Katsunori Nakamura, ${ }^{b}$ Shinji Kikuchi, ${ }^{c}$ and Shigeru Ohmori*,a \\ ${ }^{a}$ Department of Pharmacy, Shinshu University Hospital; 3-1-1 Asahi, Matsumoto 390-8621, Japan: ${ }^{b}$ Graduate \\ School of Pharmaceutical Sciences, Nagoya City University; 3-1 Tanabe-dori, Mizuho-ku, Nagoya 467-8603, \\ Japan: and ${ }^{c}$ Kissei Pharmaceutical Co., Ltd.; 4365-1 Kashiwabara, Hotaka, Azumino 399-8304, Japan. \\ Received October 3, 2012; accepted November 15, 2012; advance publication released online December 8, 2012
}

\begin{abstract}
We reported previously that monkey embryonic stem cells (ESCs) were differentiated into hepatocytes by formation of embryoid bodies (EBs). However, this EB formation method is not always efficient for assays using a large number of samples simultaneously. A dispersion culture system, one of the differentiation methods without EB formation, is able to more efficiently provide a large number of feeder-free undifferentiated cells. A previous study demonstrated the effectiveness of the Rho-associated kinase inhibitor Y-27632 for feeder-free dispersion culture and induction of differentiation of monkey ESCs into neural cells. In the present study, the induction of differentiation of cynomolgus monkey ESCs (cmESCs) into hepatocytes was performed by the dispersion culture method, and the expression and drug inducibility of cytochrome P450 (CYP) enzymes in these hepatocytes were examined. The cmESCs were successfully differentiated into hepatocytes under feeder-free dispersion culture conditions supplemented with Y-27632. The hepatocytes differentiated from cmESCs expressed the mRNAs for three hepatocyte marker genes ( $\alpha$-fetoprotein, albumin, CYP7A1) and several CYP enzymes, as measured by real-time polymerase chain reaction. In particular, the basal expression of cmCYP3A4 (3A8) in these hepatocytes was detected at mRNA and enzyme activity (testosterone $6 \beta$-hydroxylation) levels. Furthermore, the expression and activity of cmCYP3A4 (3A8) were significantly upregulated by rifampicin. These results indicated the effectiveness of Y-27632 supplementation for feeder-free dispersed culture and induction of differentiation into hepatocytes, and the expression of functional CYP enzyme(s) in cmESC-derived hepatic cells.
\end{abstract}

Key words embryonic stem cell; differentiation; hepatocyte; monkey; cytochrome P450; feeder-free dispersed culture

Investigation of drug metabolism with human hepatocytes is important in the early stages of drug development. However, primary human hepatocytes are short-lived and cannot be maintained in culture over the long term. In addition, there are large donor-dependent variations in drug metabolism. On the other hand, human embryonic stem cells (ESCs) are able to replicate infinitely and differentiate into various types of somatic cells including germ cells. ${ }^{1}$ Thus, they represent an attractive source to provide large numbers of cells that can be utilized for the development of candidate drug-screening strategies in place of primary cells. ${ }^{2}$ However, ethical and legal restrictions have limited the availability of human ESCs. The phenotype of human ESCs is known to closely resemble that of monkey ESCs but not mouse ESCs with regard to morphology, leukemia inhibitory factor responsiveness, gene expression profiles, and some disease models. ${ }^{1,3-6)}$ Thus, monkey ESCs are a more suitable model for preclinical research of drug development. In particular, hepatocytes derived from monkey ESCs may be useful for pharmacokinetic studies, such as investigation of drug-drug interactions and the inducibility of drug-metabolizing enzymes, including cytochrome P450 (CYP).

We reported previously that monkey ESCs were successfully differentiated into hepatocytes by the formation of embryoid bodies (EBs) and treatment with specific growth factors and cytokines critical for hepatic differentiation.

The authors declare no conflict of interest.
EBs can mimic the inductive microenvironment required for liver organogenesis ${ }^{8-10)}$ and develop into many different cell types in culture. However, this EB formation method is not always appropriate for assays with large numbers of samples, such as high-throughput screening, because the formation of EBs is inefficient. A dispersion culture system, one of the differentiation methods without EB formation, can more efficiently provide a large number of feeder-free undifferentiated cells. The Rho-associated kinase (ROCK) inhibitor Y-27632 enables expansion from single-cell culture of human ESCs under dispersion culture conditions because the ROCK inhibitor markedly reduces dissociation-induced apoptosis of human ESCs. ${ }^{11)}$ Furthermore, Takehara et al. ${ }^{12)}$ conducted direct neural stem cell induction from cynomolgus monkey (Macaca fascicularis) ESCs (cmESCs) using Y-27632 and demonstrated the effectiveness of Y-27632 supplementation for feeder-free culture and induction of differentiation. However, it is not clear whether this dispersion culture method is effective for differentiation of monkey ESCs into hepatocytes.

In the present study, we carried out induction of hepatocyte differentiation from cmESCs by the dispersion culture method and examined expression and drug inducibility of CYP in the differentiated cells.

\section{MATERIALS AND METHODS}

Materials Growth Factor Reduced BD Matrigel Matrix (Matrigel reduced) was obtained from BD Biosciences 
(A)

Feeder-free culture

using Matrigel reduced

cmESCs (undifferentiated cells)

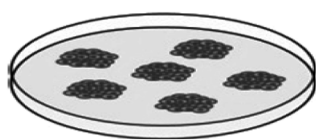

$\$ 10 \mathrm{~cm}-$ plate

Mitomycin C mitotically inactivated $\mathrm{MEF}$ (25-fold dilution) and

MEF-conditioned medium
Differentiation of cmESCs

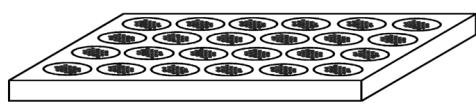

24-well plate

Matrigel reduced (25-fold dilution)

Rho-associated kinase inhibitor

(ROCK inhibitor; Y-27632)

(B)

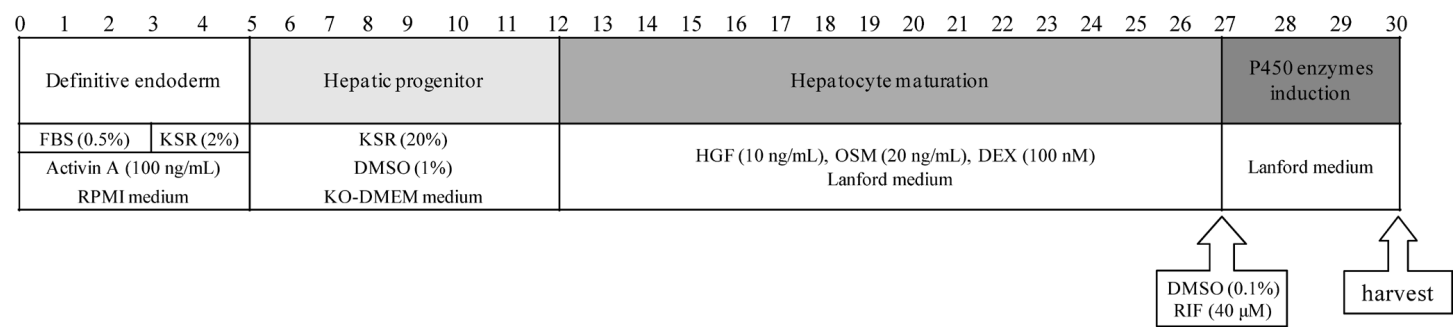

Fig. 1. Scheme of in Vitro Differentiation of cmESCs into Hepatocytes

(A) Illustration of the feeder-free dispersion culture of undifferentiated cmESCs. (B) Schematic procedure of differentiation of cmESCs into hepatocytes and drug treatment.

(Bedford, MA, U.S.A.); mitomycin C, Dulbecco's modified Eagle's medium (DMEM), William's E medium with GlutaMAX without phenol red, MEM non-essential amino acid solution $(100 \times)$, and $6 \beta$-hydroxytestosterone from Sigma (St. Louis, MO, U.S.A.); murine embryonic fibroblasts (MEF) from Oriental Yeast (Tokyo, Japan); RPMI1640 medium supplemented with GlutaMAX, KnockOut ${ }^{\mathrm{TM}}$ DMEM, KnockOut Serum Replacement (KSR), minimum essential medium (MEM), L-glutamine, $0.25 \%(\mathrm{w} / \mathrm{v})$ trypsin-ethylenediaminetetraacetic acid (EDTA), and SuperScript ${ }^{\mathrm{TM}}$ III First-Strand Synthesis System for reverse transcription-polymerase chain reaction (RT-PCR) from Invitrogen Life Technologies (Carlsbad, CA, U.S.A.); fetal bovine serum (FBS) from EquitechBio, Inc. (Kerrville, TX, U.S.A.); recombinant human activin $A$ and recombinant human hepatocyte growth factor (HGF) from Funakoshi Co., Ltd. (Tokyo, Japan); modified Lanford medium from Charles River Laboratories Japan Inc. (Yokohama, Japan); recombinant human basic fibroblast growth factor (bFGF), Y-27632, oncostatin M (OSM), dexamethasone (DEX), rifampicin (RIF), testosterone, and dimethyl sulfoxide (DMSO) from Wako Pure Chemicals (Osaka, Japan); $\left[{ }^{2} \mathrm{H}_{7}\right] 6 \beta$ hydroxytestosterone from BD Gentest (Franklin Lakes, NJ, U.S.A.); illustra RNAspin Mini RNA Isolation kit from GE Healthcare (Tokyo, Japan); SYBR ${ }^{\circledR}$ Green real-time PCR Master Mix from TaKaRa Bio (Otsu, Japan). All other reagents used were of the highest quality available.

ESC Culture and Differentiation The cmESCs (CMK6) were generously provided by Tanabe Seiyaku Co., Ltd. (Osaka, Japan) $^{4}$ and maintained according to the method reported previously ${ }^{7)}$ except that recombinant human bFGF was added to ES medium. Feeder-free dispersed culture was carried out as follows (Fig. 1A). The cmESCs were cultured in the presence of $10 \mu \mathrm{M} \mathrm{Y}-27632$ for $1 \mathrm{~h}$ before detaching the cells from the feeder layer. After detachment of the cmESCs, contaminating MEF were removed by incubating the cell suspension on gelatin-coated plates (BD Falcon, Franklin Lakes, NJ, U.S.A.) at $37^{\circ} \mathrm{C}$ for $2 \mathrm{~h}$. The cmESC clumps were recovered from the suspension by centrifugation, incubated in $0.25 \%$ (w/v) trypsin-EDTA solution at $37^{\circ} \mathrm{C}$ for $5 \mathrm{~min}$, and dissociated into single cells by pipetting. The cells were passed through a Cell Strainer ( $40 \mu \mathrm{m}$ mesh; BD Falcon) and seeded onto culture plates $6 \mathrm{~cm}$ in diameter (BD Falcon) coated with Matrigel reduced (25-fold dilution). The cmESCs were cultured in medium conditioned by contact with MEF with $4 \mathrm{ng}$ / $\mathrm{mL}$ recombinant human $\mathrm{bFGF}$ and $10 \mu \mathrm{M}$ Y-27632 for the first $24 \mathrm{~h}$. The medium was changed for MEF-conditioned medium for cmESCs without Y-27632.

When cmESCs reached approximately 70\% confluence, differentiation was initiated by replacing RPMI1640 medium supplemented with GlutaMax containing 0.5\% FBS and $100 \mathrm{ng} / \mathrm{mL}$ activin A (Fig. 1B). After $72 \mathrm{~h}$, the medium was changed to RPMI1640 medium supplemented with GlutaMax containing $2 \% \mathrm{KSR}$ and $100 \mathrm{ng} / \mathrm{mL}$ activin A, and culture was continued for $48 \mathrm{~h}$. The cells were passaged onto 24-well plates coated with Matrigel reduced (25-fold dilution) and cultured in KnockOut $^{\mathrm{TM}}$ DMEM containing 20\% KSR, $1 \mathrm{~mm}$ L-glutamine, 1\% MEM nonessential amino acids, and 1\% DMSO for $7 \mathrm{~d}$. Finally, the cells were cultured in modified Lanford medium containing $10 \mathrm{ng} / \mathrm{mL} \mathrm{HGF}, 20 \mathrm{ng} / \mathrm{mL}$ OSM, and $100 \mathrm{~nm}$ DEX. The medium was changed daily during differentiation. 
Table 1. Primers Used for Real-Time PCR Analysis

\begin{tabular}{|c|c|c|c|}
\hline Genes & Forward primer $\left(5^{\prime}-3^{\prime}\right)$ & Reverse primer $\left(5^{\prime}-3^{\prime}\right)$ & Product (bp) \\
\hline AFP & ACTATTGGCCTGTGGTGAGG & CACCCTGAGCTTGACACAGA & 224 \\
\hline ALB & CTTCCTGGGCATGTTTTTGT & GGCTCTTCCACAAGAGGTTG & 177 \\
\hline CYP1A1 & CTAGACACAGTGATTGGCAGGTC & GGTTGACCCATAGCTTCTGGTCA & 232 \\
\hline cmCYP2B6 (2B30) & GGGGCATTGAAGAAGAATGA & ATTTTGCCCACACCACTCTC & 188 \\
\hline cmCYP2C9 (2C43) & TGATTCCCAAGGGTACAACC & AAATTGCCACCTTCATCCAG & 118 \\
\hline cmCYP2D6 (2D17) & AGATCGACGACGTGATAGGG & GTCCCCTTAGGGATGAGGAA & 178 \\
\hline cmCYP3A4 (3A8) & CCAAGAAGCTTTTAAGATTTGATTTC & ATCTACTCGGTGCTTTTGTGTA & 191 \\
\hline cmCYP3A5 (3A66) & TTTGCCCAATAAGGCACCTG & GGTTGGAATCACCACCATTG & 181 \\
\hline CYP7A1 & ATTTGGTGCCAATCCTCTTG & CATCCTTTGGGTCAATGCTT & 215 \\
\hline $\mathrm{AhR}$ & ACTCCACTTCAGCCACCATC & CTCGTGCACAGTTCTGCTTC & 146 \\
\hline PXR & AAGGATGCAAGGGCTTTTTC & TTCTTCATGCCGCTCTCC & 151 \\
\hline GAPDH & GTCAGTGGACCTGACCT & TGCTGTAGCCAAATTCGTTG & 245 \\
\hline
\end{tabular}

AFP, $\alpha$-fetoprotein; ALB, albumin; AhR, aryl hydrocarbon receptor; PXR, pregnane X receptor; GADPH, glyceraldehyde-3-phosphate dehydrogenase.

Drug Treatment To clarify the effects of RIF on expression of CYP, cmESC-derived hepatocytes were treated with $40 \mu \mathrm{M}$ RIF for $72 \mathrm{~h}$ (Fig. 1B). The compound was dissolved in DMSO, which was added to the modified Lanford medium at a final concentration of $0.1 \%$.

Primary Hepatocyte Culture Primary cynomolgus monkey hepatocytes (primary cmHCs, Batch HEP 18605) were obtained from BIOPREDIC International (Renes, France). The primary cmHCs were thawed according to the manufacturer's instructions. Briefly, the primary cmHCs were cultured on 24-well plates (BD Falcon) in William's E medium with GlutaMax without phenol red for $72 \mathrm{~h}$, and the medium was changed daily.

Real-time PCR Analysis Total RNA was isolated from the cells and the liver of an adult male monkey (Ina Research Inc., Ina, Japan) using the illustra RNAspin Mini RNA Isolation kit according to the manufacturer's protocol. First-strand cDNA was generated from $5 \mu \mathrm{g}$ of total RNA. Reverse transcription reaction was performed using the SuperScript ${ }^{\mathrm{TM}}$ III First-Strand Synthesis System for RT-PCR in accordance with the manufacturer's instructions. For detection of mRNA expression levels, CYP mRNAs were analyzed by $\mathrm{SYBR}^{\circledR}$ Green real-time quantitative PCR. All PCR procedures were performed using the ABI Prism 7300 Real-time PCR System (Applied Biosystems, Foster City, CA, U.S.A.). PCR was performed in a mixture consisting of $10 \mu \mathrm{L}$ of SYBR ${ }^{\circledR}$ Green realtime PCR Master Mix, $0.4 \mu \mathrm{L}$ of $10 \mu \mathrm{M}$ forward and reverse primers, $0.4 \mu \mathrm{L}$ of dye, $7.8 \mu \mathrm{L}$ of water, and $1 \mu \mathrm{L}$ template cDNA in a total of $20 \mu \mathrm{L}$. The primers used are summarized in Table 1. The levels of these mRNAs were normalized relative to that of glyceraldehyde-3-phosphate dehydrogenase (GAPDH) mRNA.

Measurement of Cellular Activity of Testosterone 6及-Hydroxylation Following drug treatment, cmESC-derived hepatocytes were incubated with $100 \mu \mathrm{M}$ testosterone in modified Lanford medium for $6 \mathrm{~h}$. On the other hand, primary cmHCs were incubated with $100 \mu \mathrm{M}$ testosterone in MEM for $6 \mathrm{~h}$. After incubation, each medium was collected and $6 \beta$-hydroxytestosterone was measured by LC-MS/MS under the conditions described below.

Instrument An Agilent 1100 series HPLC system (Agilent Technologies, Waldbronn, Germany) consisting of a binary pump, a degasser linked to a CTC HTS PAL New Wash System Autosampler (AMR Inc., Tokyo, Japan) was used.
Table 2. Timetable for HPLC

\begin{tabular}{ccc}
\hline \hline Time $(\min )$ & Solvent A $(\%)$ & Solvent B (\%) \\
\hline 0 & 10 & 90 \\
3 & 10 & 90 \\
6 & 90 & 10 \\
9 & 90 & 10 \\
9.1 & 10 & 90 \\
16 & 10 & 90 \\
\hline
\end{tabular}

Solvents A: $10 \mathrm{~mm}$ ammonium acetate in water. Solvents B: $0.1 \%$ formic acid in methanol.

Mass spectrometry was performed on an API 4000 triple quadruple instrument (Applied Biosystems/Sciex, Foster City, CA, U.S.A.) equipped with a TurboIonSpray ${ }^{\circledR}$ electrospray ionization (ESI) interface. Data processing was performed with the Analyst 1.4.2 software package (Applied Biosystems/Sciex).

Chromatographic Conditions Chromatographic separation was performed on a reversed-phase CAPCELL PAK C18 MG III column $(50 \times 4.6 \mathrm{~mm}$ i.d., $5 \mu \mathrm{m}$; Shiseido Co., Inc., Tokyo, Japan). The column temperature was kept constant at $40^{\circ} \mathrm{C}$. The mobile phase consisted of a mixture of $10 \mathrm{~mm}$ ammonium acetate in water (A) with $0.1 \%$ formic acid in methanol (B) and was delivered at a flow rate of $0.5 \mathrm{~mL} / \mathrm{min}$. A stepwise gradient was used as shown in Table 2.

Mass Spectrometry Conditions The mass spectrometer was operated using the ESI source in positive ion mode. To optimize all of the MS parameters, standard solutions (100 ng/ $\mathrm{mL}$ ) and internal standard were infused into the mass spectrometer at a flow rate of $250 \mu \mathrm{L} / \mathrm{min}$. The ion spray voltage (IS) was set at $4500 \mathrm{~V}$. The TurboIonSpray probe temperature was maintained at $600^{\circ} \mathrm{C}$. The instrument parameters viz., nebulizer gas, curtain gas, auxiliary gas, and collision gas, were set at $60,15,80$, and 5, respectively. Compound parameters viz., declustering potential, collision energy, entrance potential, and collision exit potential, were $40,20,10$, and 15 , respectively, for $6 \beta$-hydroxytestosterone and $\left[{ }^{2} \mathrm{H}_{7}\right] 6 \beta$ hydroxytestosterone. Zero air was used as the source gas, while nitrogen was used as both curtain and collision gas. The mass spectrometer was operated in ESI positive ion mode and detection of the ions was performed in the multiple reaction monitoring (MRM) mode, monitoring the transition of $m / z 305$ precursor ion $[\mathrm{M}+\mathrm{H}]$ to the $m / z 269$ product ion for $6 \beta$-hydroxytestosterone (retention time $8.7 \mathrm{~min}$ ) and $\mathrm{m} / \mathrm{z} 312$ precursor ion $[\mathrm{M}+\mathrm{H}]$ to the $\mathrm{m} / \mathrm{z} 276$ product ion for 
(A)

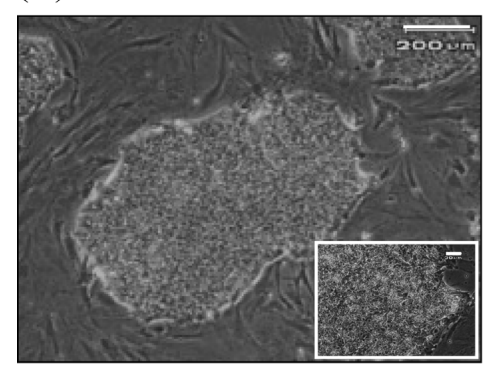

(C)

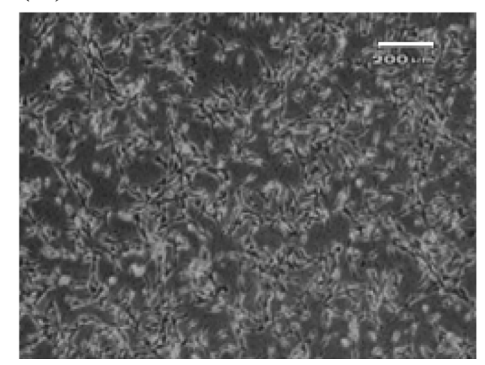

(B)

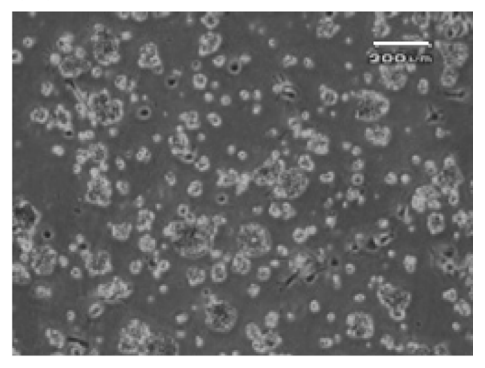

(D)

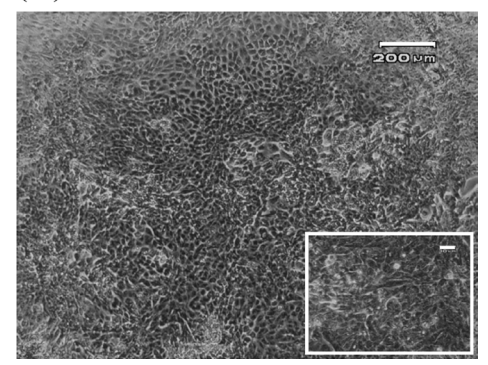

Fig. 2. Morphology of cmESCs in Individual Culture Processes

(A) Undifferentiated cmESCs. (B) Suspended cmESCs incubated on gelatin-coated plates for $2 \mathrm{~h}$. (C) cmESCs cultured on plates $6 \mathrm{~cm}$ in diameter coated with Matrigel reduced ( 25 -fold dilution) for $24 \mathrm{~h}$. (D) cmESC-derived cells at $27 \mathrm{~d}$ after the initiation of hepatocyte differentiation. The cells were visualized by phase microscopy. Bars, $200 \mu \mathrm{m} ; 30 \mu \mathrm{m}$ for A and D insets.

$\left[{ }^{2} \mathrm{H}_{7}\right] 6 \beta$-hydroxytestosterone $(8.7 \mathrm{~min})$. Quadrupoles Q1 and Q3 were set to unit resolution. Data acquisition and quantification were performed using Analyst software version 1.4.2 (Applied Biosystems, MDS Sciex, Toronto, ON, Canada).

Calibration Standards Calibration standards to cover the assay range of $10-5000 \mathrm{~nm} 6 \beta$-hydroxytestosterone were prepared by adding $10 \mu \mathrm{L}$ of $0.1,0.5,1,5,10$, and $50 \mu \mathrm{M}$ working standards to $0.1 \mathrm{~mL}$ aliquots of control reaction mixture.

Statistical Analysis Statistical significance was assessed using the unpaired $t$ test. In all analyses, $p<0.05$ was taken to indicate statistical significance.

\section{RESULTS}

Morphology of cmESCs at Individual Culture Steps A typical colony of undifferentiated cmESCs is shown in Fig. 2A. As reported previously for primate ESCs, undifferentiated cmESCs formed tightly packed and flat colonies. ${ }^{4)}$ Each cell had a high nucleus/cytoplasm ratio and prominent nucleolus. In an effort to circumvent the problem of apoptosis in cmESC culture, the single-cell dispersed culture was performed under feeder-free cell culture conditions using Y-27632. The undifferentiated cmESCs were cultured in the presence of $10 \mu \mathrm{M}$ Y-27632 for $1 \mathrm{~h}$ before detaching the cells from the feeder layer. After the cmESC colonies were dissociated by trypsin and suspended, the cells were seeded on gelatin-coated plates. In this procedure, contaminating MEF adhered to the plate bottom, whereas the cmESCs did not (Fig. 2B). The cmESC clumps were recovered from the suspension and dissociated into single cells by pipetting. The single cells were cultured on culture plates $6 \mathrm{~cm}$ in diameter coated with Matrigel reduced (25-fold dilution) in the presence of $10 \mu \mathrm{M}$ Y-27632 for first $24 \mathrm{~h}$. The cmESCs proliferated on the feeder-free culture plates (Fig. 2C). Twenty-seven days after initiation of hepatocyte differentiation, cells showed characteristic morphologies of hepatocytes, i.e., polygonal in shape and multiple nuclei (Fig. 2D). Y-27632 was effective for cmESC survival under dispersion culture conditions.

Expression of Hepatocyte Markers and CYP Enzymes in Primary cmHCs and cmESC-Derived Hepatocytes The mRNA expression levels of hepatocyte marker genes and CYP enzymes in primary cmHCs and differentiated cells from cmESCs were measured by a real-time PCR method. As shown in Fig. 3, the mRNAs of hepatocyte marker genes, $\alpha$-fetoprotein (AFP), albumin (ALB), and CYP7A1, were detected in cmESC-derived hepatocytes together with those of CYP1A1, cmCYP2B6 (2B30), cmCYP2C9 (2C43), cmCYP2D6 (2D17), cmCYP3A4 (3A8), cmCYP3A5 (3A66), pregnane $\mathrm{X}$ receptor (PXR), and aryl hydrocarbon receptor (AhR). The mRNA levels of AFP and CYP7A1 in differentiated cells from cmESCs were 16- and 21-fold, respectively, higher than those in primary cmHCs, although the expression level of ALB was approximately 80-fold lower in cmESCderived hepatocytes than in primary cmHCs. The mRNA levels of CYP1A1, cmCYP2B6 (2B30), cmCYP2C9 (2C43), cmCYP2D6 (2D17), cmCYP3A4 (3A8), cmCYP3A5 (3A66), and PXR in the cells differentiated from cmESCs were 386-, 7.4-, 284-, 1.6-, 136-, 5.9-, and 13-fold, respectively, lower than those in primary cmHCs. In contrast, the expression level of AhR mRNA in cmESC-derived hepatocytes was 2.2-fold higher than that in primary cmHCs.

Testosterone 6 $\beta$-Hydroxylase Activity of Primary cmHCs and cmESC-Derived Hepatocytes Testosterone $6 \beta$-hydroxylase activity as a marker of CYP3A, especially cmCYP3A4 (3A8), was evaluated with primary cmHCs and cmESC-derived hepatocytes. Testosterone $6 \beta$-hydroxylase activity was detected in the cells differentiated from cmESCs (Fig. 4). The activity was about one sixth that of the primary 


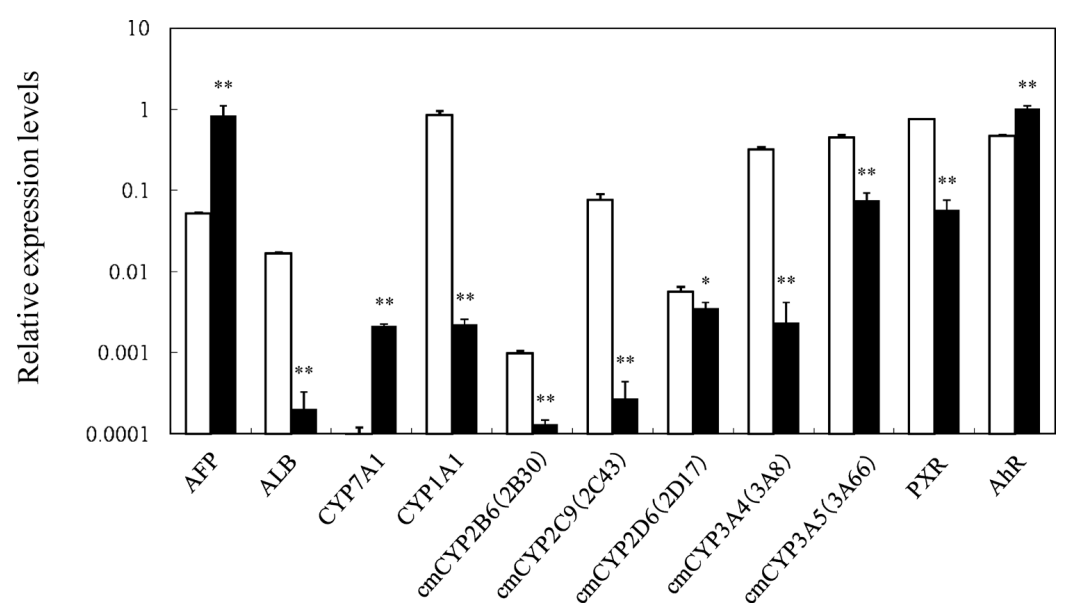

Fig. 3. Expression Levels of mRNAs for Hepatocyte Markers and CYP Enzymes in Primary cmHCs and cmESC-Derived Hepatocytes

Target mRNAs were analyzed by SYBR Green real-time PCR as described in Materials and Methods. Data are presented as the relative levels (means \pm S.D., $n=3$ ) of primary cmHCs (open columns) or cmESC-derived hepatocytes (closed columns) to adult monkey liver. Significantly different from primary $\mathrm{cmHCs}(* p<0.05, * * p<0.01$ ).

cmHCs.

Effects of RIF on the Expression Level and Activity of cmCYP3A4 (3A8) in the Cells Differentiated from cmESCs The induction potency of cmCYP3A4 (3A8) by RIF, which is known as a cmCYP3A4 (3A8) inducer, ${ }^{13,14)}$ was examined with the cells differentiated from cmESCs. The expression of cmCYP3A4 (3A8) mRNA was significantly induced by RIF in the cmESC-derived hepatocytes (Fig. 5A). The activity of testosterone $6 \beta$-hydroxylase in the cmESC-derived hepatocytes was significantly enhanced by RIF (Fig. 5B).

\section{DISCUSSION}

The method to induce differentiation of ESCs to hepatic cells was first established using mouse cells. ${ }^{15-17)}$ Many of these methods involve a procedure to differentiate ESCs by EB formation and adhesion culture. EBs differentiate into three embryonic germ layers by suspended cell culture of ESCs, and these cells can then further differentiate into multiple cell types, including hepatocytes, in vitro. ${ }^{18)}$ This method was applied to the induction of differentiation of monkey ESCs into hepatic cells. ${ }^{7)}$ In recent studies, however, a method to add some direct inducing factors to a monolayer culture system of an undifferentiated ESC colony without EB formation has been used extensively. Furthermore, some improved methods for more efficient differentiation into hepatocytes have been reported. Among these methods, the stepwise addition of two or more factors is most common. ${ }^{19-23)}$ However, if these complicated operations are not performed adequately, the death of ESCs can easily occur. In previous culture systems, remarkable reduction of the number of live cells was a technical obstacle to the induction of differentiation. Watanabe et al. ${ }^{11)}$ found that the death of human ESCs occurring after cell dissociation is triggered by activation of ROCK, and that the ROCK inhibitor Y-27632 can control the death of ESCs. Interestingly, Takehara et al. ${ }^{12)}$ demonstrated that Y-27632 also promotes survival of cmESCs and enables expansion from single cells without loss of their pluripotent characteristics. These findings suggest that reactions to the ROCK inhibitor may be preserved in primate ESCs. In addition, it has been shown that Y-27632 supplementation also enables cmESC

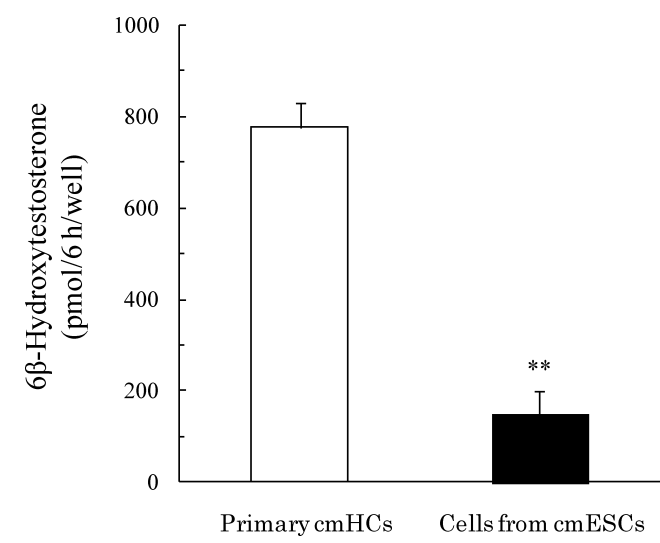

Fig. 4. Testosterone $6 \beta$-Hydroxylase Activity of Primary cmHCs and cmESC-Derived Hepatocytes

Primary cmHCs and cmESC-derived hepatocytes were incubated with testosterone at a final concentration of $100 \mu \mathrm{m}$ for $6 \mathrm{~h}$. Testosterone $6 \beta$-hydroxylase activity as a marker of CYP3A, especially cmCYP3A4 (3A8), was measured by LC-MS/MS as described in Materials and Methods. Values are expressed as the means \pm S.D. $(n=3)$. Significantly different from primary cmHCs $(* * p<0.01)$.

expansion in feeder-free culture. ${ }^{12)}$ Feeder cells supply secretory components, extracellular matrix, and intercellular contacts for maintenance of an undifferentiated state and pluripotency of ESCs. However, the use of feeder cells leads to the potential for cross-contamination, such as the passing of animal pathogens to ESCs. On the other hand, feeder-free culture is a system to maintain ESCs in an undifferentiated state without direct contact with feeder cells. The ROCK inhibitor, Y-27632, is an important factor to enable adhesion and proliferation of primate ESCs in feeder-free culture. In the present study, we confirmed that dissociated cmESCs treated with Y-27632 were protected from cell death in feeder-free culture and formed clumps (Fig. 2B). Furthermore, the cells differentiated from cmESCs showed multinuclear morphology characteristic of hepatocytes at the final stage of differentiation (Fig. 2D). This feeder-free dispersion culture method was reproducible without any technical obstacles.

Although ALB is the most abundant protein synthesized by mature hepatocytes, its expression starts in early fetal hepatocytes and reaches the maximal level in adult hepatocytes. ${ }^{24)}$ Our study showed that the expression of ALB mRNA in 
(A) cmCYP3A4 (3A8) mRNA

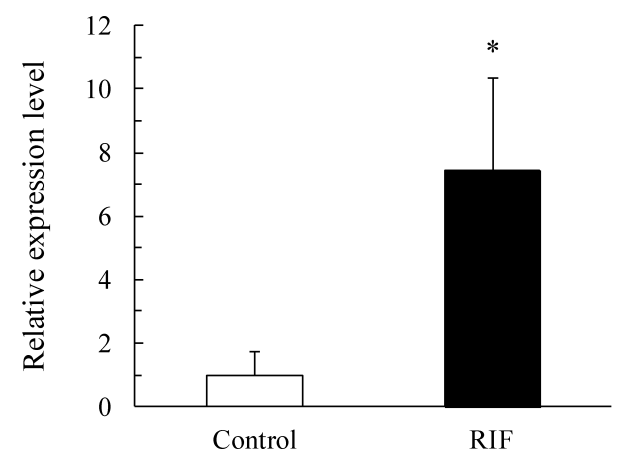

(B) Testosterone 6ß-hydroxylation

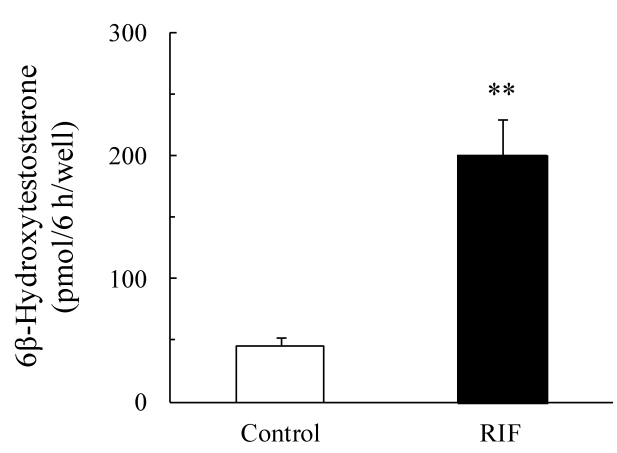

Fig. 5. Effects of RIF on cmCYP3A4 (3A8) mRNA Expression Level and Testosterone 6 $\beta$-Hydroxylase Activity in Cells Differentiated from cmESCs

cmESC-Derived hepatocytes were treated with vehicle $(0.1 \%$ DMSO) or $40 \mu \mathrm{M}$ RIF for $72 \mathrm{~h}$. After treatment with RIF, the cells were incubated with testosterone at a concentration of $100 \mu \mathrm{m}$ for $6 \mathrm{~h}$. (A) cmCYP3A4 (3A8) mRNA was analyzed by SYBR Green real-time PCR as described in Materials and Methods. Data are presented as the relative levels (means \pm S.D., $n=3$ ) of RIF treatment to vehicle treatment. (B) Testosterone $6 \beta$-hydroxylase activity was measured by LC-MS/MS as described in Materials and Methods. Significantly different from control $(* p<0.05, * * p<0.01)$.

hepatocytes differentiated from cmESCs was markedly lower than in primary cmHCs and adult monkey liver. On the other hand, AFP is a marker of endodermal differentiation as well as an early fetal hepatic marker; its expression level decreases as the liver develops into the adult phenotype. In this study, the expression of AFP mRNA in cmESC-derived hepatocytes was higher than that in primary cmHCs, although the mRNA level in the former cells was comparable to that in adult monkey liver. These results suggest that cmESC-derived hepatocytes may be differentiated into more immature cells rather than into mature cells.

CYP1A1 is a major CYP1A isoform expressed in the monkey liver, ${ }^{25)}$ in contrast to the human liver, which mainly expresses CYP1A2 but not CYP1A1. ${ }^{26}$ cmCYP2B6 (2B30), cmCYP2C9 (2C43), cmCYP2D6 (2D17), cmCYP3A4 (3A8), and cmCYP3A5 (3A66) are major CYP enzymes in the cynomolgus monkey liver. ${ }^{27)}$ These monkey CYP enzymes show a high degree of amino acid sequence identity (>90\%) with corresponding human CYP enzymes and catalyze typical drug oxidations of corresponding human CYP isoforms. ${ }^{28)}$ To characterize the expression of CYP1A1, cmCYP2B6 (2B30), cmCYP2C9 (2C43), cmCYP2D6 (2D17), cmCYP3A4 (3A8), and cmCYP3A5 (3A66) in hepatocytes differentiated from cmESCs, the basal gene expression patterns of these CYP enzymes were compared with primary cmHCs. The expression levels of the CYP enzymes examined were lower in cmESCderived hepatocytes than in primary cmHCs. The lower expression of these CYP enzymes may be associated with hepatocyte maturity. In this study, the cmESC-derived hepatic cells were found to express cmCYP3A4 (3A8) mRNA. This enzyme function in differentiated hepatocytes was confirmed by testosterone $6 \beta$-hydroxylase activity. To our knowledge, this is the first study showing the functional expression of certain CYP enzyme in cmESC-derived hepatic cells. Furthermore, these cells showed inducibility of cmCYP3A4 (3A8) by RIF at mRNA and activity levels. This inducibility was qualitatively reproducible. The effects of RIF on mRNA expression of cmCYP3A4 (3A8) in the cmESC-derived hepatic cells was consistent with the findings reported previously. ${ }^{7)}$ It was previously reported that cmCYP3A4 (3A8) is induced by RIF through the transcription factor $\mathrm{PXR} .^{29)}$ Our study showed that PXR is expressed in cmESC-derived hepatic cells. These results suggest that PXR is active in these hepatocytes. This has important implications for the application of cmESC-derived hepatic cells as an in vitro model for drug development. However, the functional significance of cmCYP3A4 (3A8) and PXR in cmESC-derived hepatocytes was limited in this study because the data were partially qualitative. Further improvements are required, particularly with regard to maturation and quantitative analysis, for use of cmESC-derived hepatocytes in drug screening.

In conclusion, cmESCs were successfully differentiated into hepatocytes under feeder-free dispersion culture conditions supplemented with Y-27632. In addition, the basal expression and drug inducibility of CYP enzymes were characterized in these hepatocytes. These results suggest that cmESC-derived hepatocytes may be used as a potential source for stable supply of hepatocytes for drug metabolism analysis, although further investigations are needed to improve this method.

Acknowledgments This work was partly supported by Grants-in-Aid from the Ministry of Education, Culture, Sports, Science and Technology of Japan (Nos. 20926015, 22926013, and 23926012). We also thank Ms. K. Aikawa for technical assistance. We are grateful to Tanabe Seiyaku Co., Ltd. (Osaka, Japan) for generously providing cmESCs (CMK6).

\section{REFERENCES}

1) Thomson JA, Itskovitz-Eldor J, Shapiro SS, Waknitz MA, Swiergiel JJ, Marshall VS, Jones JM. Embryonic stem cell lines derived from human blastocysts. Science, 282, 1145-1147 (1998).

2) Davila JC, Cezar GG, Thiede M, Strom S, Miki T, Trosko J. Use and application of stem cells in toxicology. Toxicol. Sci., 79, 214 223 (2004).

3) Reubinoff BE, Pera MF, Fong CY, Trounson A, Bongso A. Embryonic stem cell lines from human blastocysts: somatic differentiation in vitro. Nat. Biotechnol., 18, 399-404 (2000).

4) Suemori H, Tada $T$, Torii R, Hosoi $Y$, Kobayashi K, Imahie H, Kondo Y, Iritani A, Nakatsuji N. Establishment of embryonic stem 
cell lines from cynomolgus monkey blastocysts produced by IVF or ICSI. Dev. Dyn., 222, 273-279 (2001).

5) Ginis I, Luo Y, Miura T, Thies S, Brandenberger R, Gerecht-Nir S, Amit M, Hoke A, Carpenter MK, Itskovitz-Eldor J, Rao MS. Differences between human and mouse embryonic stem cells. Dev. Biol., 269, 360-380 (2004).

6) Suemori H, Nakatsuji N. Generation and characterization of monkey embryonic stem cells. Methods Mol. Biol., 329, 81-89 (2006).

7) Momose $Y$, Matsunaga $T$, Murai K, Takezawa T, Ohmori S. Differentiation of monkey embryonic stem cells into hepatocytes and mRNA expression of cytochrome p450 enzymes responsible for drug metabolism: comparison of embryoid body formation conditions and matrices. Biol. Pharm. Bull., 32, 619-626 (2009).

8) Lavon N, Benvenisty N. Study of hepatocyte differentiation using embryonic stem cells. J. Cell. Biochem., 96, 1193-1202 (2005).

9) Asahina K, Fujimori H, Shimizu-Saito K, Kumashiro Y, Okamura K, Tanaka Y, Teramoto K, Arii S, Teraoka H. Expression of the liver-specific gene Cyp7al reveals hepatic differentiation in embryoid bodies derived from mouse embryonic stem cells. Genes Cells, 9, 1297-1308 (2004).

10) Heo J, Factor VM, Uren T, Takahama Y, Lee JS, Major M, Feinstone SM, Thorgeirsson SS. Hepatic precursors derived from murine embryonic stem cells contribute to regeneration of injured liver. Hepatology, 44, 1478-1486 (2006).

11) Watanabe $K$, Ueno $M$, Kamiya $D$, Nishiyama A, Matsumura $M$, Wataya T, Takahashi JB, Nishikawa S, Nishikawa S, Muguruma K, Sasai Y. A ROCK inhibitor permits survival of dissociated human embryonic stem cells. Nat. Biotechnol., 25, 681-686 (2007).

12) Takehara $T$, Teramura $T$, Onodera $Y$, Kakegawa R, Fukunaga $N$, Takenoshita M, Sagawa N, Fukuda K, Hosoi Y. Rho-associated kinase inhibitor Y-27632 promotes survival of cynomolgus monkey embryonic stem cells. Mol. Hum. Reprod., 14, 627-634 (2008).

13) Nishimura M, Koeda A, Suganuma Y, Suzuki E, Shimizu T, Nakayama M, Satoh T, Narimatsu S, Naito S. Comparison of inducibility of CYP1A and CYP3A mRNAs by prototypical inducers in primary cultures of human, cynomolgus monkey, and rat hepatocytes. Drug Metab. Pharmacokinet., 22, 178-186 (2007).

14) Ohtsuka T, Yoshikawa T, Kozakai K, Tsuneto Y, Uno Y, Utoh M, Yamazaki H, Kume T. Alprazolam as an in vivo probe for studying induction of CYP3A in cynomolgus monkeys. Drug Metab. Dispos., 38, 1806-1813 (2010).

15) Hamazaki T, Iiboshi Y, Oka M, Papst PJ, Meacham AM, Zon LI, Terada N. Hepatic maturation in differentiating embryonic stem cells in vitro. FEBS Lett., 497, 15-19 (2001).

16) Chinzei R, Tanaka Y, Shimizu-Saito K, Hara Y, Kakinuma S, Watanabe M, Teramoto K, Arii S, Takase K, Sato C, Terada N, Teraoka H. Embryoid-body cells derived from a mouse embryonic stem cell line show differentiation into functional hepatocytes. Hepatology, 36, 22-29 (2002).

17) Maezawa $K$, Miyazato $K$, Matsunaga $T$, Momose $Y$, Imamura $T$, Johkura K, Sasaki K, Ohmori S. Expression of cytochrome P450 and transcription factors during in vitro differentiation of mouse embryonic stem cells into hepatocytes. Drug Metab. Pharmacokinet., 23, 188-195 (2008).

18) Doetschman TC, Eistetter H, Katz M, Schmidt W, Kemler R. The in vitro development of blastocyst-derived embryonic stem cell lines: formation of visceral yolk sac, blood islands and myocardium. $J$. Embryol. Exp. Morphol., 87, 27-45 (1985).

19) Cai J, Zhao Y, Liu Y, Ye F, Song Z, Qin H, Meng S, Chen Y, Zhou R, Song X, Guo Y, Ding M, Deng H. Directed differentiation of human embryonic stem cells into functional hepatic cells. Hepatology, 45, 1229-1239 (2007).

20) Agarwal S, Holton KL, Lanza R. Efficient differentiation of functional hepatocytes from human embryonic stem cells. Stem Cells, 26, 1117-1127 (2008).

21) Hay DC, Zhao D, Fletcher J, Hewitt ZA, McLean D, UrruticoecheaUriguen A, Black JR, Elcombe C, Ross JA, Wolf R, Cui W. Efficient differentiation of hepatocytes from human embryonic stem cells exhibiting markers recapitulating liver development in vivo. Stem Cells, 26, 894-902 (2008).

22) Touboul T, Hannan NR, Corbineau S, Martinez A, Martinet C, Branchereau S, Mainot S, Strick-Marchand H, Pedersen R, Di Santo J, Weber A, Vallier L. Generation of functional hepatocytes from human embryonic stem cells under chemically defined conditions that recapitulate liver development. Hepatology, 51, 1754-1765 (2010).

23) Zhou M, Li P, Tan L, Qu S, Ying QL, Song H. Differentiation of mouse embryonic stem cells into hepatocytes induced by a combination of cytokines and sodium butyrate. J. Cell. Biochem., 109, 606-614 (2010).

24) Sellem $\mathrm{CH}$, Frain M, Erdos T, Sala-Trepat JM. Differential expression of albumin and alpha-fetoprotein genes in fetal tissues of mouse and rat. Dev. Biol., 102, 51-60 (1984).

25) Sakuma T, Hieda M, Igarashi T, Ohgiya S, Nagata R, Nemoto N, Kamataki T. Molecular cloning and functional analysis of cynomolgus monkey CYP1A2. Biochem. Pharmacol., 56, 131-139 (1998).

26) Schweikl H, Taylor JA, Kitareewan S, Linko P, Nagorney D, Goldstein JA. Expression of CYP1A1 and CYP1A2 genes in human liver. Pharmacogenetics, 3, 239-249 (1993).

27) Uehara S, Murayama N, Nakanishi Y, Zeldin DC, Yamazaki H, Uno Y. Immunochemical detection of cytochrome P450 enzymes in liver microsomes of 27 cynomolgus monkeys. J. Pharmacol. Exp. Ther., 339, 654-661 (2011).

28) Iwasaki K, Uno Y. Cynomolgus monkey CYPs: a comparison with human CYPs. Xenobiotica, 39, 578-581 (2009).

29) Kim S, Dinchuk JE, Anthony MN, Orcutt T, Zoeckler ME, Sauer MB, Mosure KW, Vuppugalla R, Grace JE Jr, Simmermacher J, Dulac HA, Pizzano J, Sinz M. Evaluation of cynomolgus monkey pregnane $\mathrm{X}$ receptor, primary hepatocyte, and in vivo pharmacokinetic changes in predicting human CYP3A4 induction. Drug Metab. Dispos., 38, 16-24 (2010). 